\title{
Thyroid-Induced Toxicity of Check-Point Inhibitors Immunotherapy in the Treatment of Advanced Non-Small Cell Lung Cancer
}

\author{
Emanuela Rossi ${ }^{1}$, Assunta Sgambato ${ }^{3}$, Giovanni De Chiara ${ }^{2}$, Fortunato Ciardiello ${ }^{1}$, and Cesare Gridelli ${ }^{1 *}$ \\ ${ }^{1}$ Division of Medical Oncology, S.G. Moscati Hospital, Avellino, Italy \\ ${ }^{2}$ Division of Pathologic Anatomy, S.G. Moscati Hospital, Avellino, Italy \\ ${ }^{3}$ Department of Clinical and Experimental Medicine, Second University of Naples, Naples, Italy
}

\begin{abstract}
Received: March 24, 2016; Accepted: April 13, 2016; Published: April 17, 2016
*Corresponding author: Cesare Gridelli, Division of Medical Oncology, S.G. Moscati Hospital, Avellino, Italy, Tel: +39-0825-203-573; Fax: +39-0825203-556; E-mail: cgridelli@libero.it
\end{abstract}

\begin{abstract}
Immunotherapy represents a new strategy in the fight against cancer diseases based on the key role that immune system plays to identify and destroy foreign or abnormal cells in the body, including tumor cells. Nowadays, two immune-checkpoint inhibitors with antitumor activity have been approved for treatment of advanced nonsmall cell lung cancer (NSCLC): Nivolumab and Pembrolizumab (antiprogrammed death 1, anti-PD-1). However, these inhibitors are related to a unique class of mechanism-based toxicities, termed immunerelated Adverse Events (irAEs) that have an autoimmune etiology. Among endocrinopathies due to immuno-checkpoint antibodies, the thyreopathies are frequently reported, probably because antithyroid autoimmunity represents the most common autoimmune disease in humans. The spectrum of thyreopathies reported in NSCLC patients during therapy with immune-checkpoint inhibitors includes: thyroiditis, primary and secondary hypothyroidism and hyperthyroidism. In view of the ongoing increased employment of these drugs in the treatment of NSCLC, irAEs, described in selected patients in clinical trials, will began more frequent and with different clinical impact in routine practice. This manuscript focuses particularly on thyroid disorders induced by immunotherapies employed in NSCLC, in order to suggest the strategies for identify, diagnose and treat them without affecting patient outcomes.
\end{abstract}

Keywords: Cytotoxic T-lymphocyte-associated antigen-4 Hyperthyroidism; Hypothyroidism; Immune-checkpoint inhibitors; Immune-related adverse events; Non-small-cell lung cancer; Pogrammed cell death protein-1; Thyroiditis

\section{Introduction}

Different strategies have been developed over time to improve the poor survival of patients affected by advanced NonSmall Cell Lung Cancer (NSCLC): radiotherapy, chemotherapy and molecular targeted therapies. Recently, in view of the hypothesis that the immunosuppression could be involved in the mechanism of carcinogenesis, by allowing tumor cells to evade immune-surveillance, new immunological drugs have been synthesized [1,2]. In particular, discovery of immune checkpoints, receptors expressed on $\mathrm{T}$ cells that regulate immune response, has led to development of monoclonal antibodies against these molecules that alter $\mathrm{T}$ cell functions and determine antitumor activity [3]. Antibodies blocking Cytotoxic T-LymphocyteAssociated Antigen 4 (CTLA-4), and blocking the Programmed Death 1 (PD-1) receptor and his ligands (PD-L1 and PD-L2) have recently shown impressive response rates in the management of some solid tumors, particularly in melanoma, NSCLC, renal cell carcinoma, bladder cancer and ovarian cancer [3,4]. These promising drugs were first approved for metastatic melanoma and recently are available for lung cancer. Immune checkpoints inhibitor nivolumab (BMS-936558) has been approved by Food and Drug Administration (FDA) and European Medicines Agency (EMA) for the treatment of advanced pretreated NSCLC patients, while pembrolizumab (MK-3475, anti-PD-1) by FDA always in the second-line setting but only for patients with tumor strongly expressing ( $>50 \%$ ) PD-L1. Other immune checkpoint inhibitors tested in NSCLC patients are atezolizumab (MPDL3280A) durvalumab (MEDI4736), and BMS-936559 among antiPD-L1 and ipilimumab and tremelimumab among anti-CTLA-4 antibodies. However, different immune checkpoint inhibitors and their combinations have been tested in ongoing clinical trials for lung cancer patients [5].

Although these immunological checkpoint-blocking antibodies are revolutionizing cancer therapeutics, a unique class of mechanism-based toxicities, termed immune-related Adverse Events (irAEs), has emerged [6]. These toxicities have an autoimmune aetiology and are very different from toxicities observed with conventional cytotoxic chemotherapy. Unlike cytotoxic agents, whose side effects are mainly expression of the block of rapidly dividing cells, immune-checkpoint inhibitors, by altering immune-surveillance, have often side effects associated with autoimmune diseases. These irAEs include: dermatological and gastrointestinal events, hepatitis, pneumonitis, uveitis, pancreatitis, and endocrinopathies [6]. Among endocrinopathies due to immuno-checkpoint antibodies, the thyreopathies are frequently reported [7], probably because anti-thyroid 
autoimmunity is the most common autoimmune disease in humans. Furthermore, in view of the ongoing increased employment of these drugs in the treatment of NSCLC, the incidence of their toxicities reported in clinical trials will turn into more frequent clinical cases in routine practice. This manuscript focuses particularly on thyroid disorders induced by immunocheckpoint inhibitors employed in NSCLC, in order to suggest the strategies for identify, diagnose and treat them without affecting patient outcomes.

\section{Pathogenesis of Thyroid Autoimmunity}

In the spectrum of autoimmune diseases, thyroid autoimmunity is one of the few conditions for which the autoantigens have been unequivocally identified and are known to play a role in disease pathogenesis. Most importantly, the Thyroid-Stimulating Hormone Receptor (TSHR), Thyroid Peroxidase (TPO), and Thyroglobulin (Tg) carry within themselves unusual and sometimes unique characteristics, as size, abundance, membrane association, glycosylation, and polymorphisms, that play critical roles in the breakdown of self-tolerance leading to thyroid autoimmunity [8].

$\mathrm{Tg}$ is the largest and most abundant thyroid autoantigen in the thyroid: is a soluble molecule that undergoes iodination, needed for the function of $\mathrm{Tg}$ as a prohormone of Tetraiodothyronine (T4) and Triiodothyronine (T3), but not required for recognition by human autoantibodies [9].

TPO is the primary enzyme involved in thyroid hormone synthesis: is a membrane-bound homodimer of two subunits with a heme prosthetic group, essential for enzymatic activity but not involved in TPO autoantibody recognition [10].

The TSHR is a member of the rhodopsin-like family of G protein-coupled receptors. Accumulating observations for human TSHR autoantibodies indicate that the TSHR A-subunit, rather than the membrane-bound receptor, is the main autoantigen. Thyroid-Stimulating Antibodies (TSAbs) that activate the TSHR are the direct cause of Graves' hyperthyroidism [11]. Autoimmunity to TPO and/or Tg is associated with lymphocytic infiltration of the thyroid and sometimes with hypothyroidism [12]. In clinical practice, many patients, particularly women, have autoantibodies to TPO and/or Tg but do not progress to hypothyroidism [13]. Autopsy studies have demonstrated the presence of thyroid lymphocytic infiltration associated with the presence in serum of thyroid autoantibodies in the absence of manifest clinical disease [14]. The important implication of this association is that TPO and Tg autoantibodies in euthyroid individuals likely reflect a subclinical disease [15]. Consequently, the term "Hashimoto's Thyroiditis" (HT) refers to individuals with thyroid autoantibodies regardless of their thyroid function status. In this disease, autoimmunity to Tg and/or TPO rises gradually over many years and thyroid reserve is maintained for the tropic effects of TSH, until it is ultimately overcome by massive lymphocytic infiltration, fibrosis, and thyroid follicle damage, causing overt hypothyroidism. However, the destruction of at least $90 \%$ of the thyroid tissue is required for causing overt hypothyroidism. In contrast, TSAbs, which are potent at low concentrations [16], induce hyperthyroidism at an early stage in the autoimmune response.

Graves' disease and HT taken together have a high prevalence, making autoimmunity to the thyroid gland the most common autoimmune disease affecting humans. These diseases arise in genetically susceptible individuals, for the loss of tolerance to thyroid antigens in association with environmental factors, including iodine uptake, radiation, smoking, selenium, drugs, environmental toxins, and micro-organisms [8]. Furthermore, there is evidence that infection with hepatitis $C$ virus plays a primary role in inducing autoimmune thyroiditis [17].

The recent advent of immunotherapy among the therapeutic options in the treatment of advanced NSCLC, causing the loss of immune tolerance to thyroid antigens, is an immune intervention that frequently leads to the development of thyroid autoimmunity.

\section{Immunotherapy of NSCLC}

The immune system plays a critical role in identifying and destroying foreign or abnormal cells in the body, including suppressing tumor growth [18]. Immune-surveillance involves both innate and adaptive immune mechanisms, including Natural Killer (NK) cells, CD8+ cytotoxic T-lymphocytes, CD4+ T-helper cells, and macrophages.

Antitumor immune-response is a multi-step process starting from naive T-cells which circulate in blood through lymph nodes and recognize foreign ("nonself") antigens presented by specific Antigen-Presenting Cells (APC), typically dendritic cells, through the major histocompatibility complex class I. This step alone is inadequate for successful T-cell activation and immune destruction of cancer cells because co-regulatory signals, either inhibitory or stimulatory, are also required $[3,19]$. This intricate counterbalance of co-stimulatory and co-inhibitory cell-to-cell signals between various components of the immune system regulates immune responses, whether against tumor cells or infected cells, and prevents damage against healthy tissues.

CTLA-4 and PD-1 pathways are two examples of immunecheckpoint pathways involved in controlling T-cell immune responses. PD-1 and CTLA- 4 receptors are similar in structure, both expressed on T cells (PD-1 can be expressed also on B cells or NK cells), but they bind distinct ligands.

\section{CTLA-4 and anti-CTLA-4 antibodies}

CTLA-4 is expressed on surface of T-cells and regulates the amplitude of T-cell activation, reduces $\mathrm{T}$ helper cell function and stimulates regulatory $\mathrm{T}$ (Tregs) cell immunosuppressive activity [1]. This is a transmembrane glycoprotein with considerable homology to CD28 (a stimulatory checkpoint) which binds to same ligands B7-1 (CD80) and B7-2 (CD86) on the surface of APCs and leads to T-cell proliferation. Once a cytotoxic $\mathrm{T}$ cell becomes active, it expresses CTLA-4 on its cell surface, which then competes with the co-stimulatory molecule CD28 for their mutually shared ligands on the APC. However, unlike CD28, CTLA4 inhibits T-cell responses and is important for maintenance of immune tolerance.

Notably, CTLA-4 was the first non-HLA (Human Leukocyte 
Antigen system) association identified for Graves' disease [20] and is one of 10 confirmed Graves' disease loci of immunesusceptibility $[21,22]$. Indeed, CTLA-4 gene polymorphisms have been associated with autoimmune thyroid diseases including Graves' disease and Hashimoto's thyroiditis [23,24]. It has been hypothesized that TSHR antigen is presented to the T-Cell Receptor (TCR) by the APC cells, leading to the development of potentially autoreactive $\mathrm{T}$ cells. The interaction of $\mathrm{B} 7$ (on the APC) and CTLA-4 leads to a decrease of the Tymphocyte, largely by a decrease of the TCR; the block of this interaction, caused by anti-CTLA-4 antibodies, prevents the down-regulation of T cells and could lead to the autoimmune process that causes Grave's disease [20].

Ipilimumab, a fully humanized Ig G1 monoclonal antibody anti-CTLA-4, binds to CTLA-4 and blocks its interaction with B7 molecules, preventing $\mathrm{T}$ cell inactivation. Ipilimumab, already approved for metastatic melanoma, has shown promise in one phase II trial in 204 NSCLC chemo-naïve patients. The irAEs described in this study were milder than those seen when ipilimumab was used as single agent for treatment of melanoma, probably due to the steroid administration concomitant to chemotherapy in NSCLC. In the spectrum of irAEs, one case each of G3 hypophysitis and G3 hypopituitarism with central hypothyroidism was observed in concurrent ipilimumab arm, causing treatment discontinuation [25]. Generally, these toxicities linked to CTLA-4 inhibitors tend to occur on first 12 weeks of therapy, but, in rare cases, can also occur in the weeks and even months following discontinuation of therapy [26].

\section{PD-1 and anti PD-1/ anti PD-L1}

PD-1 (CD279) receptor is an inhibitory checkpoint expressed on the surface of activated T cells, B cells, NK cells, dendritic cells, macrophages, and host tissues that plays an important role in the balance of tumor immunity and inflammatory reactions [3]. PD-1 binds to one of its ligands, typically PD-L1 (or B7-H1 or CD274) or PD-L2 (or B7-DC or CD273) expressed in tumor cells and APC, leading to T-cell inactivation. Unlike CTLA-4, PD-1 acts within peripheral tissues, inhibiting T-cell signaling, cytotoxic activity, proliferation, survival and effector function of $\mathrm{T}$ cells and also promoting differentiation of CD4+ T cells into T regulatory cells and inducing $\mathrm{T}$ cell apoptosis. So, CTLA- 4 regulates T-cell activity at an early stage, limiting initiation of a T-cell response to self-antigens in the lymph nodes and preventing autoimmunity, whereas PD-1 predominantly regulates later effector T-cell activity within tumor microenvironment [27].

Interruption of PD-1: PD-L1 and PD-1: PD-L2 binding by antiPD-1 antibodies or interruption of PD-1:PD-L1 binding by antiPD-L1 antibodies restores T-cell immune responses.

Currently, there are two PD-1 inhibitors available for lung cancer: nivolumab for treatment of patients with advanced NSCLC in progression during or after platinum-based chemotherapy (approved by FDA and EMA), pembrolizumab by FDA for treatment of patients whose NSCLC strongly expresses PD-L1 (> 50\%) and whose disease worsened during or after platinumbased chemotherapy.
Nivolumab (BMS-936558) is a fully human IgG4 antibody blocking the PD-1 receptor. In a phase Ib trial enrolling patients affected by different cancers, including 75 NSCLC patients, nivolumab was administered biweekly at escalating doses ranging from 0.3 to $10 \mathrm{mg} / \mathrm{kg}$. IrAEs of any-grade were observed in $39 \%$ of the patients including any-grade hypothyroidism in $3 \%$ of total population ( $1 \%$ at dose of $3 \mathrm{mg} / \mathrm{kg}$ and $5 \%$ at $10 \mathrm{mg} / \mathrm{kg}$ ) [28]. In 129 heavily pretreated advanced NSCLC, nivolumab showed an overall objective response rate (ORR) of $17 \%$ across all doses $17 \%$ and $18 \%$ in squamous and nonsquamous histologies, respectively) in another phase I trial. Endocrinopathies were reported in $6.2 \%$ of patients [29]. Two phase III trials compared nivolumab with docetaxel in 272 pretreated squamous (CheckMate 017) [30] and 582 nonsquamous (CheckMate 057) [31] NSCLC patients reporting a significant better OS. In CheckMate 017 trial, hypothyroidism was reported in $4 \%$ of patients (no cases of grade 3-4 hypothyroidism) [30]. In CheckMate 057 trial, grade 1-2 hypothyroidism, including thyroiditis, occurred in $7 \%(20 / 287)$ of patients treated with nivolumab and $0 \%(0 / 268)$ in docetaxel arm, while elevated TSH occurred in $17 \%$ and $5 \%$ of patients, respectively. The median time to onset of hypothyroidism/ thyroiditis was 2.9 months. All 20 patients received levothyroxine: two patients received corticosteroids (one of whom received high-dose corticosteroids). Complete resolution of hypothyroidism occurred in one patient. There was a temporarily withheld due to hypothyroidism/ thyroiditis in three patients (no cases of discontinuation). Grade 1-2 hyperthyroidism occurred in $1.4 \%$ (4/287) of patients. The median time to onset was 2 months. Two of four patients received methimazole and one patient also received treatment with high-dose corticosteroids, with complete resolution [31]. The frequencies of treatment-related AEs, serious adverse events, and adverse events leading to discontinuation of the study drug were similar regardless PD-L1 expression level (1\% or higher and less than 1\%) [31].

CheckMate 012 is an ongoing multi-arm phase $1 \mathrm{~b}$ trial evaluating nivolumab alone and in various combinations, such as platinum-based chemotherapy, erlotinib, bevacizumab, and ipilimumab in patients with chemotherapy-naïve advanced NSCLC (NCT01454102).

Nivolumab combined with ipilimumab mirrored the dose and schedules used in metastatic melanoma. All grade hypothyroidism was reported in $14 \%$ of patients in combined arm compared to $9 \%$ in ipilimumab as single-agent in advanced melanoma (no grade 3-4 hypothyroidism reported). In NSCLC patients, response rates with these combinations were $13 \%$ and $20 \%$, respectively. However, the toxicity was more than that seen with single-agent nivolumab and more than we have observed with the combination in melanoma. Therefore several new dosing schedules for this combination are under evaluation in chemo-naïve 148 patients. All of the dosing regimens showed clinical activity, reporting a confirmed ORR of $13 \%$ to $39 \%$ and an "encouraging" median PFS of 4.9 to 10.6 months. Tumors expressing PD-L1 had better responses than those whose tumors did not express PD-L1. A lower frequency of treatment-related grade 3/4 AEs leading to discontinuation (3-10\%) was reported. 


\section{Endocrine toxicity was observed in 3-8\% of patients [32].}

Pembrolizumab (MK-3475) is a humanized IgG4 monoclonal antibody against PD-1 that demonstrated a robust antitumor activity in both treatment naïve and pretreated advanced NSCLC patients $[33,34]$. Pembrolizumab was tested in a large, multicohort, phase 1b KEYNOTE-001 study enrolling 495 naïve and pretreated NSCLC patients, treated with various doses and regimens of the anti-PD-1 inhibitor. Pembrolizumab showed an acceptable side-effect profile and produced durable responses, regardless dosed used. Hypothyroidism was observed in $6.9 \%$ of patients (one case of severe G3-G5 hypothyroidism). The median time to onset was 4.2 months, and the median duration was 5.8 months. No patients discontinued pembrolizumab due to hypothyroidism and all cases were successfully treated with medical therapy. Among all the patients, ORR was 19.4\%, with median PFS of 3.7 months, and median OS of 12.0 months. A PDL1 tumour proportion score of $50 \%$ or greater was associated with better outcomes [35]. In KEYNOTE-010 trial pembrolizumab - $2 \mathrm{mg} / \mathrm{kg}$ or $10 \mathrm{mg} / \mathrm{kg}$ every 3 weeks - prolongs OS in 1034 PDL1-positive NSCLC that progressed after at least platinum-based chemotherapy and has a favorable benefit-to-risk profile. Among irAEs, the most common thyreopathies were: hypothyroidism ( $8 \%$ at both doses), hyperthyroidism ( $4 \%$ at dose of $2 \mathrm{mg} / \mathrm{kg}$ and $6 \%$ at $10 \mathrm{mg} / \mathrm{kg}$, with one case of severe G3-G5 hyperthyroidism), and thyroiditis (1\% at $2 \mathrm{mg} / \mathrm{kg}$ dose) [36].

Atezolizumab (MPDL3280A) is an anti-PD-L1 monoclonal antibody, Immunoglobulin G (IgG) type with Fc domain engineered modification to avoid antibody-dependent cell mediated cytotoxicity (ADCC). In a phase I study, MPDL3280A, at dose of $1200 \mathrm{mg}$ IV every 3 weeks, led to antitumor responses, with an ORR of $23 \%$ in pretreated patients with NSCLC [37]. Phase II POPLAR study showed that atezolizumab significantly improved OS compared to docetaxel as second line treatment (median OS: 12.6 versus 9.7 months in the overall patient population; HR: $0.73 ; P=0.040$ ). An increased efficacy of atezolizumab was observed with increasing PD-L1 expression on tumor cells and/or tumor-infiltrating immune cells. Among AEs, hypothyroidism was reported in $5 \%$ of patients [38].

Durvalumab (MEDI 4736) is a human IgG1 mAb against PDL1, with an engineered Fc domain to prevent ADCC. A phase I/II trial evaluated its safety and clinical activity in NSCLC reporting durable responses and higher ORR in squamous NSCLC $(21 \%$ versus $10 \%$ in non-squamous) and PD-L1 positive (23\%) patients. Hyperthyroidism was reported in $4 \%$ of patients, and in one case of these it was of grade 3-4 [39].

\section{Diagnosis and Managment of Thyreopathies caused by Immune Checkpoints}

The spectrum of thyreopathies caused by immune checkpoint inhibitors includes: thyroiditis, primary and secondary hypothyroidism and hyperthyroidism. The incidence of hyperthyroidism has been lower than thyroiditis and hypothyroidism for both CTLA-4 and PD-1/PD-L1 inhibition [40]. The clinical presentation of thyreopathies by immune checkpoints is sometimes unusual in patients with NSCLC, often old and frail patients with complications from prior therapies. It follows that the diagnosis could be made more difficult by their insidious clinical appearance, with symptoms that can be wrongly confused with a progression of disease. Furthermore, because of this insidious and nonspecific clinical picture, the incidence of thyreopathies due to immuno-checkpoints reported in clinical trials may have been underestimated. The time of onset of thyreopathies due to immune checkpoint inhibitors ranged from 0,7 weeks to 19 months from initiation of therapy. Although the dosage of thyroid auto-antibodies was not systematically carried out in all the trials that have used inhibitors of the immune checkpoint in patients with NSCLC, the thyreopathies seen in these trials were likely expression of the development of thyroid autoimmunity [40].

\section{Thyroiditis}

Autoimmune thyroiditis, also called HT, is the most common form of thyroiditis described in patients with NSCLC during therapy with inhibitors of immune checkpoints $[4,28]$. Often patients are asymptomatic and the clinical course of chronic thyroiditis is usually characterized by a slow progression to overt hypothyroidism. In a rare form of thyroiditis, known as silent thyroiditis or painless thyroiditis, there is the appearance of thyrotoxicosis, linked to the release of thyroid hormones by the destroyed thyroid. Thyrotoxicosis is generally transient and is characterized by symptoms caused by toxic action of iodothyronine at the tissue level: sweating, tremor, palpitations, dyspnea, diarrhea etc. The phase of thyrotoxicosis is characterized by the rise of Free Triiodothyronine (FT3), Free Tetraiodothyronine (FT4) and Tg, released from damaged follicles, with TSH suppression. Scintigraphy, therefore, reveals a reduced uptake by the thyroid both for the damage of thyrocytes, and for the failure of stimulation by TSH [41]. In a recent study, ten cases of thyroiditis were described in patients treated with anti-PD- 1 antibodies for metastatic malignancies. Six patients presented with transient thyrotoxicosis that required temporary beta-blocker therapy and had spontaneous resolution with subsequent hypothyroidism. In 4 of them (67\%) anti-TPO and anti-Thyroglobulin (anti-Tg) antibodies were documented. Four patients presented with hypothyroidism, without a previously documented thyrotoxic phase, occurring 6-8 weeks after initial drug exposure. All of these patients had positive antithyroid antibodies and required thyroid hormone replacement therapy for a minimum of 6 months [42]. It has been hypothesized that, as thyroid autoimmunity stimulated during treatment with antiCTLA-4 antibodies could be linked to polymorphic variations of the CTLA-4 receptor gene [18], so a similar condition of polymorphic variants of the PD-1 receptor gene, may account for the occurrence of thyroiditis in a subgroup of patients treated with anti-PD-1 antibodies [42].

High anti-TPO Abs and anti-Tg Abs were also reported after two cycles of ipilimumab therapy in patients treated for advanced melanoma [43].

The diagnosis of thyroiditis is based on laboratory data indicating the presence of high levels of antithyroid antibodies, 
more frequently anti-TPO, associated with thyroid function that can be extremely variable according to the stage of the disease: normal thyroid function, transient hyperthyroidism, subclinical hypothyroidism, clinical hypothyroidism. Ultrasound examination may be helpful in highlighting an inflammatory context, hypoechoic and inhomogeneous.

During therapy with anti-immunocheckpoints antibodies, routine measurements of thyroid autoantibodies, will better clarify whether thyroiditis related to these drugs, have an autoimmune pathogenesis, so closely resembling HT.

In many cases, no treatment is required because the patient is often asymptomatic, with TSH levels in the standard range. Substitution treatment with Levotiroxine (L-T4) is required in case of overt hypothyroidism and in case of subclinical hypothyroidism with TSH $>10 \mathrm{mUI} / \mathrm{L}$ (see below). Although this is an autoimmune disease, steroid treatment is not recommended because of side effects and because it is not associated with a real therapeutic advantage [41]. During thyrotoxicosis phase, orally beta-blockers may be used, while thyrostatic substances are absolutely useless as thyrotoxicosis is not supported by an increased thyroid hormone production but by the release of preformed thyroid hormones contained in the follicles destroyed by the inflammatory process. However, with appropriate therapy for thyroid disfunction, the patients that have thyroiditis as adverse immune events linked to immune checkpoint inhibitors, can safety continue anticancer therapy.

\section{Hypothyroidism}

Hypothyroidism, characterized by insufficient production of thyroid hormones, has been frequently reported in NSCLC patients among irAEs due to immune-checkpoints antibodies. Generally, it occurs after 2-4 infusions of anti-CTLA-4, while the timing of onset with PD-1/PDL1 has not been well established [6]. Improved cancer survival and clinical response to treatment with immunotherapies mainly used in the past, as interleukin-2, has been noted in patients with different cancers. The presence of hypothyroidism, in these instances, is a marker of remission, improved treatment response, and decreased tumor growth $[44,45]$. Primary hypothyroidism is supported by a thyroid disease, mostly an autoimmune thyroiditis that causes a reduced secretion of thyroid hormones with consequent compensatory increase in TSH. From a clinical-laboratory point of view it can be distinguished a subclinical hypothyroidism, characterized by high serum concentration of TSH in the presence of normal levels of FT3 and FT4 with soft or no signs and symptoms of hypothyroidism and an overt hypothyroidism, characterized by high serum concentration of TSH in the presence of low levels of FT3 and FT4 with clear signs and symptoms of hypothyroidism [41]. The symptoms of hypothyroidism vary, depending on the severity of hormone deficiency; they include: fatigue, constipation, lethargy, bradycardia, loss of appetite, weight gain, eyelid edema, puffy face, dry skin, depression. Furthermore, among hematologic abnormalities, a macrocytic anemia and an increased risk of bleeding for a state of hypocoagulability have been reported in the forms of autoimmune hypothyroidism [41]. Because these symptoms can easily be attributed to the initial malignancy, to chemotherapy or radiation therapy, or to cancer recurrence, in NSCLC patients treated with immune-checkpoints inhibitors it is easy to miss the diagnosis of hypothyroidism. A form of severe primary hypothyroidism associated with rhabdomyolysis, with TSH $=187 \mathrm{mUI} / \mathrm{L}$ and very low FT4, has been described as irAEs of pembrolizumab, a fully human monoclonal antibody against PD1 [46]. This patient, affected by mucosal melanoma, was also treated previously with ipilimumab and remained euthyroid for 43 weeks after completing ipilimumab. Then, his severe hypothyroidism could be due to pembrolizumab therapy or a combined effect of ipilimumab followed by pembrolizumab. Furthermore, it is likely that the rhabdomyolysis is a consequence of hypothyroidism and is directly associated with the severe and acute onset of hypothyroidism [46].

Secondary hypothyroidism is supported by a reduced secretion of TSH from anterior pituitary with consequent reduced TSH stimulation of thyroid hormone production. The differential diagnosis between the two forms of hypothyroidism is made possible because in the secondary form TSH is low instead of having the compensatory increase observed in the primitive form. Secondary hypothyroidism has been described as consequence of the immune-related hypophysitis, reported in NSCLC patients both in course of nivolumab and ipilimumab, although at lower incidence and of milder grade than that observed in melanoma studies.

A probable mechanism to explain the development of secondary hypothyroidism due to immune-related hypophysitis has been reported in a study testing repeated injections of a CTLA- 4 blocking antibody in mice. The study reports that CTLA4 is expressed in pituitary endocrine cells and predominantly in TSH-secreting cells. The blocking of CTLA-4 by administration of a specific monoclonal antibody leads to pituitary infiltration and antibody formation. The study also confirmed that patients receiving ipilimumab developed pituitary antibodies [47].

The incidence of secondary hypothyroidism due to hypophysitis ranges from 1-6\% with single agent anti-PD-1/ PD-L1 mAb therapy and $2-10 \%$ in selected combination studies $[7,48]$. In NSCLC patients treated with ipilimumab, the lesser severity of hypophysitis clinical course may be due to the use of corticosteroids concomitant to chemotherapy in these patients [25]. The clinical picture of secondary hypothyroidism is that of the overt hypothyroidism, just described, but with deficiency of both thyroid hormones FT3, FT4 and TSH, without increase of anti-thyroid antibodies [49]. Furthermore, other than symptoms linked to hypothyroidism, the clinical picture could be complicated by mass effect symptoms, such as headache, asthenia, nausea, vomiting, vertigo and more rarely visual disturbances with diplopia, linked to enlargement of pituitary. These symptoms generally appear after about 11 weeks of starting therapy and require imaging studies as MRI of the brain in sellar region for a differential diagnosis with brain metastases. The clinical course of secondary hypothyroidism due to hypophysitis could be further associated to symptoms and signs of other pituitary hormonal deficiencies: more often pituitary-adrenal axis and 
pituitary-gonadal axis are involved. However, the recovery of the thyroid axis has been described in $37-50 \%$ of cases in selected studies [49].

The hypothyroidism treatment is medical and it aims at the restoration of the euthyroid state, which is achieved by administration of L-T4, regardless of the cause (primary or secondary). Generally, subclinical hypothyroidism does not require any treatment, even if $\mathrm{TSH}$ levels $>10 \mathrm{mUI} / \mathrm{L}$ have been considered critical for significantly increased risk of cardiovascular mortality and morbidity and have been stated as threshold for starting L-T4 therapy [50]. In the primary hypothyroidism L-T4 treatment is modulated on TSH levels and should bring the TSH values between 1 and $2 \mathrm{mUI} / \mathrm{L}$. In patients without heart diseases and other severe co-morbidities, it is possible starting with replacement dose of L-T4, approximately $1.6 \mathrm{mcg} / \mathrm{kg}$ body weight daily. Instead, in older and frail patients and in presence of heart diseases, L-T4 should be started at low dose, usually 12 to $25 \mathrm{mcg}$ daily and increased slowly, every 4 weeks.

In table 1 the grading of primary hypothyroidism due to immune-checkpoint blockade and the adapted algorithm for its diagnosis and management are reported [7].

In cases of secondary hypothyroidism, where TSH levels are low, the effectiveness of L-T4 therapy is evaluated clinically and by measurement of serum concentration of FT4 and FT3; the measurement of TSH does not have any meaning. Furthermore, the possibility of other concomitant hormonal deficiency must be always kept in mind, since the introduction of the substitution therapy with L-T4 in patients with secondary adrenal insufficiency may precipitate an acute adrenal crisis.

In conclusion, it is recommended to check thyroid and pituitary function prior and during treatment with immune checkpoint inhibitors and, in case of both primary and secondary hypothyroidism, appropriate L-T4 replacement can be added, without discontinuation immunotherapy. In case of severe forms (G3-G4) of hypothyroidism, the interruption of immunotherapy may be needed for short periods, until L-T4 therapy restores a clinical and hormonal euthyroid state.

\section{Hyperthyroidism}

Hyperthyroidism, unlike thyrotoxicosis due to the release of thyroid hormones by a damaged thyroid, is that condition in which the increase of thyroid hormones is linked to their increased production by hyperstimulated thyroid. Both thyrotoxicosis and hyperthyroidism have been reported among irAEs observed during therapy with immune checkpoint inhibitors [7]. These two conditions, almost indistinguishable from the clinical point of view, have different causes and also different therapies. The most common cause of hyperthyroidism observed during immune checkpoint inhibitors is Graves' disease, an autoimmune disease due to production of TSAbs that activate the TSHR [11,51]. High TSAbs were reported in 3\% of patients treated with antiPD-1 antibody nivolumab for different advanced solid cancers [4]. The most frequent symptoms due to the increased thyroid hormones (both for thyrotoxicosis and for hyperthyroidism) are tachycardia, arrhythmia, nervousness, palpitations, sweating, heat intolerance, muscle fatigue, diarrhea and weight loss despite increased appetite [41].

In patients with subclinical hyperthyroidism, these symptoms may be less severe and interesting few organs and systems. Signs and symptoms that must induce a suspicion of hyperthyroidism are weight loss, atrial fibrillation, and myopathy. It is estimated that the subclinical hyperthyroidism, defined as normal levels of FT3 and FT4 with suppressed TSH values, is associated with an increased incidence of atrial fibrillation in the elderly and is responsible for $3-13 \%$ of atrial fibrillation newly diagnosed [52].

Grave's disease is often associated with ocular disorders (burning, photophobia, exophthalmos) that give the clinical framework of Grave's ophthalmopathy (GO). A case of thyroidlike orbitopathy was described during ipilimumab therapy in a woman with normal levels of thyroid hormones, euthyroid, but with an ophthalmopathy similar to that seen in Graves' disease, sustained by TSAbs [53].

The diagnosis of hyperthyroidism is carried out by measuring TSH that is very low (suppressed) and FT3 and FT4 which are normal (subclinical hyperthyroidism) or variably higher than normal (overt hyperthyroidism). These biochemical results are in common with thyrotoxicosis due to thyroiditis. In order to better define the pathogenesis of a clinical and biochemical picture of thyrotoxicosis that appears during therapy with immune checkpoint inhibitors may be useful to dose anti-thyroid antibodies. A high level of anti-TPO Abs could be associated to both conditions, while the increase of TSAbs, that activate the TSHR, is the direct cause of Graves' hyperthyroidism. However, the differential diagnosis is possible through the execution of the thyroid scan, although in NSCLC patients during immune therapy this diagnostic procedure is not a priority. Indeed, in thyroiditis scintigraphy reveals a reduced uptake by the damaged thyroid, while in Graves' disease a high uptake is observed for the hyperfunction of thyreocytes [41].

In all cases of hyperthyroidism, symptomatic drugs, like beta-blockers, can be used for reducing symptoms. In case of Graves' hyperthyroidism the most suitable therapy includes the use of thyrostatic drugs, i.e., thionamides or propylthiouracil that reduce the activity of the thyroid. Generally, the therapy with thionamides can start with doses of attack (i.e. metimazolo 20-30 mg/day orally) that can be reduced after 4-6 weeks to maintenance doses (5-15 mg/day), monitoring clinical signs and hormonal dosages. In case of intolerance to thionamides, propylthiouracil at attack dose of $200-300 \mathrm{mg} /$ day orally then reduced to maintenance dose of 50-150 mg/day, can be used.

During thyreostatic therapy, frequent clinical and hormonal controls for the dose adjustments must be programmed [41]. In some NSCLC patients developing subclinical hyperthyroidism with constantly suppressed TSH values and appearance of soft signs and symptoms, thyreostatic therapy (other than betablockers) may be useful. In table 2 the grading of hyperthyroidism due to immune-checkpoint blockade and the adapted algorithm for its diagnosis and management are reported [7]. 
Table 1: Adapted management algorithm for primary hypothyroidism with immune checkpoint blockade.

\begin{tabular}{|l|l|l|l|l|}
\hline Grade & Clinical & Hormonal Tests & Managment & Follow-up \\
\hline 1 & Asymptomatic or little symptomatic & $\begin{array}{l}\text { FT3, FT4 normal } \\
\text { TSH > 10 mUI/L anti-TPO and } \\
\text { anti-TG usually high }\end{array}$ & Levothyroxine therapy & TSH before each cycle \\
\hline 2 & $\begin{array}{l}\text { Mild symptoms } \\
\text { fatigue, constipation, weight gain, loss of } \\
\text { appetite, dry skin, eyelid edema, puffy face }\end{array}$ & $\begin{array}{l}\text { Low FT4 and/or FT3 } \\
\text { TSH > 10 mUI/L } \\
\text { anti-TPO and anti-TG } \\
\text { usually high }\end{array}$ & Levothyroxine therapy & TSH FT4 FT3 before each cycle \\
\hline $3-4$ & $\begin{array}{l}\text { Moderate-severe symptoms: } \\
\text { Bradycardia, hypotension pericardial } \\
\text { effusion, depression, hypoventilation, stupor, } \\
\text { lethargy to mixedema coma }\end{array}$ & $\begin{array}{l}\text { Very Low FT4, FT3 } \\
\text { TSH very high anti-TPO and } \\
\text { anti-TG high }\end{array}$ & $\begin{array}{l}\text { Hospitalization } \\
\text { Levothyroxine therapy } \\
\text { Supportive therapy for } \\
\text { severe cardio-respiratory } \\
\text { symptoms } \\
\text { Discontinuation of } \\
\text { immunotherapy }\end{array}$ & $\begin{array}{l}\text { Monthly TSH FT3, FT4 } \\
\text { If improves to Grade 1 } \\
\text { consider restarting } \\
\text { immunotherapy with } \\
\text { additional steroid therapy }\end{array}$ \\
\hline
\end{tabular}

Anti-TG: Antithyroglobulin Antibody; Anti-TPO: Anti-Thyroid Peroxidase Antibodies; FT3: Free Triiodothyronine; FT4: Free Tetraiodothyronine; TSH: Thyroid-Stimulating Hormone.

Table 2: Adapted management algorithm for hyperthyroidism with immune checkpoint blockade.

\begin{tabular}{|c|c|c|c|c|}
\hline Grade & Clinical & Hormonal Tests & Managment & Follow-up \\
\hline 1 & Asymptomatic $^{*}$ & $\begin{array}{l}\text { FT3, FT4 normal Suppressed TSH } \\
\text { (<0,3 mUI/L) anti-TPO } \\
\text { and/ or anti-TG normal or high }\end{array}$ & $\begin{array}{l}\text { Avoid iodized salt. Avoid } \\
\text { mouthwashes, toothpastes and } \\
\text { any drugs containing iodine }\end{array}$ & $\begin{array}{l}\text { TSH, FT3, FT4 before each } \\
\text { cycle }\end{array}$ \\
\hline 2 & $\begin{array}{l}\text { Mild symptoms } \\
\text { weight loss, increased appetite, } \\
\text { anxiety and irritability, } \\
\text { muscle weakness, } \\
\text { menstrual irregularities, } \\
\text { fatigue, tachycardia }\end{array}$ & $\begin{array}{l}\text { Suppressed TSH (<0,1 mUI/L) } \\
\text { High FT4 and/ or FT3 anti-TPO } \\
\text { and/or anti-TG variable (usually } \\
\text { high) TSAbs receptor high, in case } \\
\text { of Grave's Disease }\end{array}$ & $\begin{array}{l}\text { Beta-blockers Steroids } \\
\text { Anti-thyroid therapy } \\
\text { with Thionamides or } \\
\text { Propylthiouracil (in cases of } \\
\text { Grave's Disease) }\end{array}$ & $\begin{array}{l}\text { TSH, FT3, FT4 before each } \\
\text { cycle }\end{array}$ \\
\hline $3-4$ & $\begin{array}{l}\text { Moderate-severe symptoms: } \\
\text { arrhythmia, atrial fibrillation, tremor, } \\
\text { sweating, insomnia, diarrhea }\end{array}$ & $\begin{array}{l}\text { Suppressed TSH }(<0,1 \mathrm{mUI} / \mathrm{L}) \\
\text { FT4, FT3 high anti-TPO and anti-TG } \\
\text { high TSAbs receptor high in case of } \\
\text { Grave's Disease }\end{array}$ & $\begin{array}{l}\text { Hospitalization Beta-blockers } \\
\text { Steroids Anti-thyroid } \\
\text { therapy with Thionamides } \\
\text { or Propylthiouracil } \\
\text { Discontinuation of } \\
\text { immunotherapy }\end{array}$ & $\begin{array}{l}\text { Monthly TSH, FT3, FT4 } \\
\text { If improves to Grade } \\
1 \text { consider restarting } \\
\text { immunotherapy with } \\
\text { additional steroid therapy }\end{array}$ \\
\hline
\end{tabular}

Anti-TG: Antithyroglobulin Antibody; Anti-TPO: Anti-Thyroid Peroxidase Antibodies; FT3: Free Triiodothyronine; FT4: Free Tetraiodothyronine;

TSAbs: Thyroid-Stimulating Antibodies; TSH: Thyroid-Stimulating Hormone.

${ }^{*}$ Pay attention to weigh loss, myopathy and atrial fibrillation, particularly in the elderly

Table 3: Hormonal levels in thyreopaties due to immune-checkpoints inhibitors.

\begin{tabular}{|l|l|l|l|l|}
\hline & FT3/FT4 & TSH & $\begin{array}{l}\text { ANTI-TPO } \\
\text { and/or ANTI-TG }\end{array}$ & TSAbs \\
\hline Euthyroid Thyroiditis & Normal & Normal & High & Normal \\
\hline Subclinical Hypothyroidism & Normal & High & High & Normal \\
\hline Primary Hypothyroidism & Low & High & High & Normal \\
\hline Secondary Hypothyroidism & Low & Low & Normal & Normal \\
\hline Subclinical Hyperthyroidism & Normal & Low & Normal/High & High \\
\hline Overt Hyperthyroidism* & High & Low & Normal/High & High \\
\hline
\end{tabular}

*In case of hyperthyroidism due to thyrotoxicosis: Reduced Uptake at Thyroid Scan

*In case of hyperthyroidism due to Grave's disease: Increased Uptake at Thyroid Scan

Anti-TG: Antithyroglobulin Antibody; Anti-TPO: Anti-Thyroid Peroxidase Antibodies; FT3: Free Triiodothyronine; FT4: Free Tetraiodothyronine;

TSAbs: Thyroid-Stimulating Antibodies; TSH: Thyroid-Stimulating Hormone.

Citation: Gridelli C, Rossi E, De Chiara G, Ciardiello F, Sgambato A (2016) Thyroid-Induced Toxicity of Check-Point Inhibitors 


\begin{tabular}{|c|c|c|c|c|c|}
\hline & L-T4 Therapy & Beta-blockers & Thyreostatic & Steroids & Management \\
\hline Euthyroid Thyroiditis & NO & NO & NO & NO & $\begin{array}{l}\text { Basal TSH, FT3, FT4, anti-TPO, anti-TG } \\
\text { TSH before each cycle }\end{array}$ \\
\hline $\begin{array}{l}\text { Subclinical } \\
\text { Hypothyroidism }\end{array}$ & if $\mathrm{TSH}>10$ & NO & NO & NO & $\begin{array}{l}\text { Basal TSH, FT3, FT4, anti-TPO, anti-TG } \\
\text { TSH, FT3, FT4 before each cycle }\end{array}$ \\
\hline $\begin{array}{l}\text { Overt Primary } \\
\text { Hypothyroidism }\end{array}$ & YES & NO & NO & NO & $\begin{array}{l}\text { Basal TSH, FT3, FT4, anti-TPO, anti-TG } \\
\text { TSH, FT3, FT4 before each cycle }\end{array}$ \\
\hline $\begin{array}{l}\text { Overt Secondary } \\
\text { Hypothyroidism }\end{array}$ & YES & NO & NO & $\begin{array}{l}\text { In case of mass } \\
\text { effect symptoms } \\
\text { and in case } \\
\text { of adrenal } \\
\text { insufficiency }\end{array}$ & $\begin{array}{l}\text { Basal CLU on 24-hour urine, TSH, } \\
\text { FT3, FT4, PRL, LH, FSH, ACTH and } \\
\text { cortisol FT3, FT4 and other lacking } \\
\text { hormones before each cycle }\end{array}$ \\
\hline $\begin{array}{l}\text { Subclinical } \\
\text { Hyperthyroidism }\end{array}$ & NO & If mild symptoms & NO & NO & $\begin{array}{l}\text { Basal TSH, FT3, FT4, anti-Tg,anti- } \\
\text { TPO, TSAbs TSH, FT3, FT4 before } \\
\text { each cycle. Avoid iodized salt and } \\
\text { mouthwashes, toothpastes and any } \\
\text { drugs containing iodine }\end{array}$ \\
\hline Overt Hyperthyroidism & NO & $\begin{array}{l}\text { In first phase, until } \\
\text { thyreostatic effect } \\
\text { of therapy has been } \\
\text { obtained }\end{array}$ & YES & $\begin{array}{l}\text { In G3-G4 } \\
\text { hyperthyroidism } \\
\text { and in severe forms } \\
\text { of GO }\end{array}$ & $\begin{array}{l}\text { Basal TSH, FT3, FT4, TSAbs anti-Tg, } \\
\text { anti-TPO TSH, FT3, FT4 before each } \\
\text { cycle Selenium could be useful in GO. } \\
\text { Avoid iodized salt and mouthwashes, } \\
\text { toothpastes and any drugs containing } \\
\text { iodine }\end{array}$ \\
\hline
\end{tabular}

ACTH: Adrenocorticotropic Hormone; Anti-TG: Antithyroglobulin Antibody; Anti-TPO: Anti-Thyroid Peroxidase Antibodies CLU: Levels of Urinary Free Cortisol; FSH: Follicle-Stimulating Hormone; FT3: Free Triiodothyronine; FT4: Free Tetraiodothyronine; GO: Grave's Ophthalmopathy LH: Luteinizing Hormone; L-T4: Levotiroxine; PRL: Prolactin; TSAbs: Thyroid-Stimulating Antibodies; TSH: Thyroid-Stimulating Hormone.

The use of high dose of intravenous glucocorticoids is indicated only in G3-G4 hyperthyroidism and in severe forms of GO with recent onset of diplopia, optic neuropathy, and proptosis [54].

Selenium levels are reported to be low in the sera of patients with newly diagnosed autoimmune thyroid conditions, particularly Graves' disease. Supplementation with selenium, an antioxidant and immunomodulatory mineral salt, is indicated in mild-moderate forms of GO, since it has been demonstrated that it determines a lowering of anti-thyroid antibody levels and slows the clinical progression of ophthalmopathy, with improvement of quality of life [54-56].

In table 3 the panels of hormonal levels useful for differential diagnosis of thyreopaties due to immune-checkpoints inhibitors have been reported.

In table 4 the principles of treatment and management of thyreopaties due to inhibitors of immune-checkpoints are summarized.

\section{Conclusion}

Immune therapy is a promising new treatment for a long-term cure of metastatic NSCLC. On the other hand, this therapy is characterized by the emergency of atypical toxicities that are likely a direct result of breaking immune tolerance. Unfortunately, prophylactic steroids and other preventive strategies to avoid them have not shown clinical benefit to date. Among endocrine side-effects, the thyroid toxicity is prevalent with the use of PD-1 and PD-L1 inhibitors, while hypophysitis, with secondary hypothyroidism, is more frequent in the case of therapy with anti-CTLA-4. Although these immune therapies have shown good activity and tolerability in patients enrolled in clinical trials, when these therapies will be extended also to unselected patients, thyroid toxicities with most clinical impact could appear. Nowadays, the time of resolution of this toxicity has not been stated because in many cases the consequent thyroid dysfunction may be permanent and timelessly managed with medical treatment. Furthermore, multiple trials are ongoing in order to examine the efficacy of combination of two different immune checkpoint modulators. The greater effectiveness of these combinations should be linked to a strengthening of autoimmunity between the two different classes of drug. On the other hand, this exacerbated autoimmunity may also cause an increased incidence of side effects with potential immunologic etiology. For this reason, before starting therapy with antiimmune checkpoints, oncologists must check thyroid function and must identify potential risk factors that could favour the emergency of autoimmune thyreopaties, like personal and family history of autoimmune disease. Furthermore, patientphysician communication and early diagnosis of immune-related thyreopathies are also emerging as critical issues to successfully manage them. However, these thyreopathies are generally mild and manageable following specific treatment guidelines and, unlike other irAEs, often permit patients to continue receiving anticancer immune-therapies from which significant clinical benefits may derive.

\section{References}

1. Dong H, Strome SE, Salomao DR, Tamura H, Hirano F, Flies DB, et al. Tumor-associated B7-H1 promotes T-cell apoptosis: a potential mechanism of immune evasion. Nat Med. 2002;8(8):793-800. 
2. Anagnostou VK, Brahmer JR. Cancer immunotherapy: a future paradigm shift in the treatment of non-small cell lung cancer. Clin Cancer Res. 2015;21(5):976-84.doi: 10.1158/1078-0432.CCR-141187.

3. Pardoll DM. The blockade of immune checkpoints in cancer immunotherapy. Nat Rev Cancer. 2012;12(4):252-64. doi: 10.1038/ $\operatorname{nrc3239.}$

4. Topalian SL, Hodi FS, Brahmer JR, Gettinger SN, Smith DC, McDermott DF, et al. Safety, activity, and immune correlates of anti-PD-1 antibody in cancer. N Engl J Med. 2012;366:2443-2454.

5. Bustamante Alvarez JG, González-Cao M, Karachaliou N, Santarpia M Viteri S, Teixidò C, et al. Advances in immunotherapy for treatment of lung cancer. Cancer Biol Med. 2015;12(3):209-22. doi: 10.7497/j. issn.2095-3941.2015.0032.

6. Chow LQM. Exploring novel immune-related toxicities and endpoints with immune- checkpoint inhibitors in Non-small-cell lung cancer. ASCO Educational Book. 2013;e280-e286. doi: 10.1200/EdBook AM.2013.33.e280.

7. Rossi E, Sgambato A, De Chiara G, Casaluce F, Losanno T, Sacco PC, et al. Endocrinopathies induced by immune-checkpoint inhibitors in advanced non-small cell lung cancer. Expert Rev Clin Pharmacol. 2016;9(3):419-28. doi: 10.1586/17512433.2016.1133289.

8. Tomer Y, Huber A. The etiology of autoimmune thyroid disease: a story of genes and environment. J Autoimmun. 2009;32(3-4):231239. doi: 10.1016/j.jaut.2009.02.007.

9. Nye L, Pontes de Carvalho LC, Roitt IM. Restrictions in the response to autologous thyroglobulin in the human. Clin Exp Immunol. 1980;41(2):252-263.

10. McLachlan SM, Rapoport B. Thyroid peroxidase as an autoantigen. Thyroid. 2007;17:939-948.

11. Rapoport B, McLachlan SM. The thyrotropin receptor in Graves disease. Thyroid. 2007;17:911-922.

12. Rapoport B, McLachlan SM. Thyroid autoimmunity. J Clin Invest. 2001;108(9):1253-1259.

13. Prentice LM, Phillips DI, Sarsero D, Beever K, McLachlan SM, Smith BR. Geographical distribution of subclinical autoimmune thyroid disease in Britain: a study using highly sensitive direct assays for autoantibodies to thyroglobulin and thyroid peroxidase. Acta Endocrinol (Copenh). 1990;123(5):493-498.

14. Yoshida H, Amino N, Yagawa K, Uemura K, Satoh M, Miyai K, et al. Association of serum antithyroid antibodies with lymphocytic infiltration of the thyroid gland: Studies of seventy autopsied cases. J Clin Endocrinol Metab. 1978;46(6):859-862.

15. Acar T, Ozbek SS, Erdogan M, Ozgen AG, Demirel SO. US findings in euthyroid patients with positive antithyroid autoantibody tests compared to normal and hypothyroid cases. Diagn Interv Radiol. 2013;19(4):265-270. doi: 10.5152/dir.2013.041.

16. de Forteza R, Smith CU, Amin J, McKenzie JM, Zakarija M. Visualization of the thyrotropin receptor on the cell surface by potent autoantibodies. J Clin Endocrinol Metab. 1994;78(5):1271-1273.

17. Tomer $Y$. Hepatitis $C$ and interferon induced thyroiditis. J Autoimmun. 2010;34:J322-J326. doi: 10.1016/j.jaut.2009.11.008.

18. Swann JB, Smyth MJ. Immune surveillance of tumors. J Clin Invest. 2007;117:1137-1146.

19. Bour-Jordan H, Esensten JH, Martinez-Llordella M, Penaranda C,
Stumpf M, Bluestone JA. Intrinsic and extrinsic control of peripheral T-cell tolerance by costimulatory molecules of the CD28/ B7 family. Immunol Rev. 2011;241(1):180-205. doi: 10.1111/j.1600065X.2011.01011.x.

20. Yanagawa T, Hidaka Y, Guimaraes V, Soliman M, DeGroot LJ. CTLA4 gene polymorphism associated with Graves' disease in a Caucasian population. J Clin Endocrinol Metab. 1995;80:41-45.

21. Brand OJ, Gough SC. Immunogenetic mechanisms leading to thyroid autoimmunity: recent advances in identifying susceptibility genes and regions. Curr Genomics. 2011;12:526-541. doi: $10.2174 / 138920211798120790$.

22. Płoski R, Szymański K, Bednarczuk T. The genetic basis of graves' disease. Curr Genomics. 2011;12(8):542-563. doi: 10.2174/138920211798120772.

23. Chistiakov DA, Turakulov RI. CTLA-4 and its role in autoimmune thyroid disease. J Mol Endocrinol. 2003;31(1):21-36.

24. Kavvoura FK, Akamizu T, Awata T, Ban Y, Chistiakov DA, Frydecka I, et al. Cytotoxic T-lymphocyte associated antigen 4 gene polymorphisms and autoimmune thyroid disease: a meta-analysis. J Clin Endocrinol Metab. 2007;92:3162-3170.

25. Lynch TJ, Bondarenko I, Luft A, Serwatowski P, Barlesi F, Chacko R, et al. Ipilimumab in combination with paclitaxel and carboplatin as firstline treatment in stage IIIB/IV non-small-cell lung cancer: results from a randomized, double-blind, multicenter phase II study. J Clin Oncol. 2012;30(17):2046-2054. doi: 10.1200/JCO.2011.38.4032.

26. Fecher LA, Agarwala SS, Hodi FS, Weber JS. Ipilimumab and its toxicities: a multidisciplinary approach. Oncologist. 2013;18(6):73343. doi: 10.1634/theoncologist.2012-0483.

27. Butte MJ, Keir ME, Phamduy TB, Sharpe AH, Freeman GJ. Programmed death-1 ligand 1 interacts specifically with the B7-1 costimulatory molecule to inhibit T cell responses. Immunity. 2007;27(1):111-122.

28. Brahmer JR, Tykodi SS, Chow LQ, Hwu WJ, Topalian SL, Hwu P, et al. Safety and activity of anti-PD-L1 antibody in patients with advanced cancer. N Engl J Med. 2012;366(26):2455-2465. doi: 10.1056/ NEJMoa1200694.

29. Gettinger SN, Horn L, Gandhi L, Spigel DR, Antonia SJ, Rizvi NA, et al. Overall survival and long-term safety of nivolumab (Antiprogrammed death 1 antibody, BMS-936558, ONO-4538) in patients with previously treated advanced non-small-cell lung cancer. J Clin Oncol. 2015;33:2004-2012. doi: 10.1200/JC0.2014.58.3708.

30. Brahmer J, Reckamp KL, Baas P, Crino L, Eberhardt WE, Poddubskaya E, et al. Nivolumab versus docetaxel in advanced squamous-cell non-small-cell lung cancer. N Engl J Med. 2015;373(2):123-135. doi: 10.1056/NEJMoa1504627.

31. Borghaei H, Paz-Ares L, Horn L, Spigel DR, Steins M, Ready NE, et al. Nivolumab versus Docetaxel in Advanced Nonsquamous Non-SmallCell Lung Cancer. N Engl J Med. 2015;22;373(17):1627-39. doi: 10.1056/NEJMoa1507643.

32. Rizvi NA, Gettinger SN, Goldman JW, Hellmann MD, Chow LQ, Juergens $\mathrm{R}$, et al. Safety and efficacy of first-line nivolumab and ipilimumab in non-small cell lung cancer. 16th World Conference on Lung Cancer. Abstract ORAL02.05. Presented September 7, 2015.

33. Garon EB, Leighl NB, Rizvi NA, Blumenschein GR, Balmanoukian AS, Eder JP et al. Safety and clinical activity of MK-3475 in previously treated patients (pts) with non-small cell lung cancer (NSCLC). J Clin Oncol. 2014;32:5s (suppl; abstr 8020). 
34. Rizvi NA, Garon EB, Patnaik A, Gandhi L, Leighl NB, Balmanoukian AS, et al. Safety and clinical activity of MK-3475 as initial therapy in patients with advanced non-small cell lung cancer (NSCLC). J Clin Oncol. 2014; 32:5 suppl; abstr 8007.

35. Garon EB, Rizvi NA, Hui R, Leighl N, Balmanoukian AS, Eder JP, et al. Pembrolizumab for the treatment of non-small-cell lung cancer. N Engl J Med. 2015;372:2018-28. doi: 10.1056/NEJMoa1501824.

36. Herbst RS, Baas P, Kim D-W, Felip E, Pérez-Gracia JL, Han J-H, et al. Pembrolizumab versus docetaxel for previously treated, PD-L1positive, advanced non-small-cell lung cancer (KEYNOTE-010): a randomised controlled trial. Lancet. 2015;S0140-6736(15):01281-7. doi: 10.1016/S0140-6736(15)01281-7.

37. Soria JC, Cruz C, Bahleda R, Delord JP, Horn L, Herbst RS, et al. Clinica activity, safety, and biomarkers of a PD-L1 blockade in non-small cell lung cancer (NSCLC): additional analyses from a clinical study of the engineered antibody MPDL3280A (anti-PDL1). Eur J Cancer. 2013 49:abstr 3408.

38. Vansteenkiste J, Fehrenbacher L, Spira AI, Mazieres J, Park K, Smith D, et al. Atezolizumab monotherapy vs docetaxel in 2L/3L non-small cell lung cancer: Primary analyses for efficacy, safety and predictive biomarkers from a randomized phase II study (POPLAR). European Cancer Congress 2015, Abstract 14LBA.

39. Rizvi NA, Brahmer JR, Ou S-HI, Segal NH, Khleif S, Hwu W-J, et al. Safety and clinical activity of MEDI4736, an anti-programmed cell deathligand 1 (PD-L1) antibody, in patients with non-small cell lung cancer (NSCLC). J Clin Oncol. 2015;33 (suppl; abstr 8032).

40. Villadolid J, Amin A. Immune checkpoint inhibitors in clinical practice: update on management of immune-related toxicities. Transl Lung Cancer Res. 2015;4(5):560-575.

41. Melmed S, Polonsky KS, Larsen, PR. Williams textbook of endocrinology. 12th ed. Philadelphia (PA): Elsevier. 2011; ISBN: 9781-4377-0324-5

42. Orlov S, Salari F, Kashat L, Walfish PG. Induction of painless thyroiditis in patients receiving programmed death 1 receptor immunotherapy for metastatic malignancies. J Clin Endocrinol Metab. 2015;May;100(5):1738-41. doi: 10.1210/jc.2014-4560.

43. Min L, Vaidya A, Becker C. Thyroid autoimmunity and ophthalmopathy related to melanoma biological therapy. Eur J Endocrinol 2011;164:303-7. doi: 10.1530/EJE-10-0833.

44. Phan GQ, Attia P, Steinberg SM White DE, Rosenberg SA. Factors associated with response to high-dose interleukin-2 in patients with metastatic melanoma. J Clin Oncol. 2001;19:3477-3482.

45. Weijl NI, Van der Harst D, Brand A, Kooy Y, Van Luxemburg S, Schroder
J, et al. Hypothyroidism during immunotherapy with interleukin-2 is associated with antithyroid antibodies and response to treatment. J Clin Oncol. 1993;11:1376-1383.

46. Min L, Hodi FS. Anti-PD1 following Ipilimumab for mucosal melanoma: durable tumor response associated with severe hypothyroidism and rhabdomyolysis. Cancer Immunol Res. 2014;2(1):15-18.

47. Iwama S, De Remigis A, Callahan MK, Slovin SF, Wolchok JD, and Caturegli P. Pituitary Expression of CTLA-4 Mediates Hypophysitis Secondary to Administration of CTLA-4 Blocking Antobody. 2014;Sci. Transl. Med. 6(230):230ra45. doi: 10.1126/scitranslmed.3008002.

48. Dillard T, Yedinak CG, Alumkal J, Fleseriu M. Anti-CTLA-4 antibody therapy associated autoimmune hypophysitis: serious immune related adverse events across a spectrum of cancer subtypes. Pituitary 2010;13:29-38. doi: 10.1007/s11102-009-0193-z.

49. Mahzari M, Liu D, Arnaout A, Lochnan H. Immune checkpoint inhibitor therapy associated hypophysitis. Clin Med Insights Endocrinol Diabetes. 2015;8:21-28. doi: 10.4137/CMED.S22469.

50. Jonklaas J, Bianco AC, Bauer AJ, Burman KD, Cappola AR, Celi FS, et al. Guidelines for the treatment of hypothyroidism: prepared by the American thyroid association task force on thyroid hormone replacement. Thyroid. 2014;24:1670-1751. doi: 10.1089/ thy.2014.0028.

51. Borodic G, Hinkle DM, Cia Y. Drug-induced graves disease from CTLA4 receptor suppression. Ophthal Plast Reconstr Surg. 2011;27(4): e87-88. doi: 10.1097/IOP.0b013e3181ef72a1.

52. Auer J, Scheibner P, Mische T, Langsteger W, Eber O, Eber B. Subclinical hyperthyroidism as a risk factor for atrial fibrillation. Am Heart J 2001;142(5):838.

53. McElnea E, Ní Mhéalóid A, Moran S, Kelly R, Fulcher T. Thyroid-like ophthalmopathy in a euthyroid patient receiving Ipilimumab. Orbit. 2014;33(6):424-7. doi: 10.3109/01676830.2014.949792.

54. Bartalena L, Marcocci C, Pinchera A. Treating severe Graves' ophthalmopathy. Bailliere's Clinics in Endocrinology and Metabolism. 1997;11(3):521-536.

55. Bartalena L, Macchia PE, Marcocci C, Salvi M, Vermiglio F. Effects of treatment modalities for Graves ' hyperthyroidism on Graves' orbitopathy: a 2015 Italian Society of Endocrinology Consensus Statement. J Endocrinol Invest. 2015;38(4):481-7. doi: 10.1007/ s40618-015-0257-z.

56. Duntas LH. Selenium and the thyroid: a close-knit connection. J Clin Endocrinol Metab. 2010;95(12):5180-5188. doi: 10.1210/jc.20100191 